Neurol Med Chir (Tokyo) 51, 136 140, 2011

\title{
Isolated Intracranial Rosai-Dorfman Disease Without Dural Attachment
} -Case Report-

\author{
Takao FUKUSHIMA, ${ }^{1}$ Kazunari YACHI, ${ }^{1}$ Akiyoshi OGINO, ${ }^{1}$ Takashi OHTA, ${ }^{1}$ \\ Takao WATANABE, ${ }^{1}$ Atsuo Yoshino, ${ }^{1}$ and Yoichi KATAYAMA ${ }^{1}$ \\ ${ }^{1}$ Department of Neurological Surgery, Nihon University School of Medicine, Tokyo
}

\begin{abstract}
A 33-year-old female presented with an isolated well-enhanced intracerebral lesion with peritumoral edema in the frontal lobe, which was tentatively diagnosed preoperatively as either a primary intraparenchymal neoplasm or metastatic brain tumor. However, histological examinations yielded a diagnosis of Rosai-Dorfman disease. Isolated intracranial Rosai-Dorfman disease is very rare, and without dural attachment, as in our case, is exceptional. The present case mimicked intraparenchymal neoplasm. Rosai-Dorfman disease should be considered in the differential diagnosis of isolated intraparenchymal tumors. Magnetic resonance imaging including diffusion-weighted imaging may be helpful in the diagnosis of isolated intracranial Rosai-Dorfman disease.
\end{abstract}

Key words: dural attachment, intraparenchymal tumor, malignant lymphoma, magnetic resonance imaging, Rosai-Dorfman disease

\section{Introduction}

Sinus histiocytosis with massive lymphadenopathy, or Rosai-Dorfman disease, is a rare but idiopathic histioproliferative disorder characterized by massive but painless cervical lymphadenopathy, fever, weight loss, and increased erythrocyte sedimentation rate. ${ }^{22)}$ Involvement of an extranodal location has been observed in about $40 \%$ of reported patients with Rosai-Dorfman disease,

Received March 17, 2010;

Accepted July 20, 2010 most commonly in the skin, respiratory tract, and bone, whereas intracranial localization is very rare. ${ }^{4}$ Most cases of Rosai-Dorfman disease with intracranial involvement show dural-based solitary or multiple masses. We treated a patient with extranodal Rosai-Dorfman disease presenting as a solitary intraparenchymal frontal lobe lesion, thought preoperatively to be either intraparenchymal neoplasm such as a malignant glioma, malignant lymphoma, or metastatic brain tumor, which had mimicked meningioma, unlike most previously reported cases. 


\section{Case Report}

A 33-year-old female presented with a one-month history of headache. Her medical history was unremarkable except for left optic neuritis that was treated at age 25 years. She had no history of focal neurological deficits or seizures, unknown pyrexia or symptoms resembling flu, or nasal obstruction/epistaxis. She had normal vital signs without cutaneous abnormalities, lymphadenopathy, or hepatosplenomegaly. Neurological examinations also revealed no abnormality.

Routine hematological and biochemical examinations
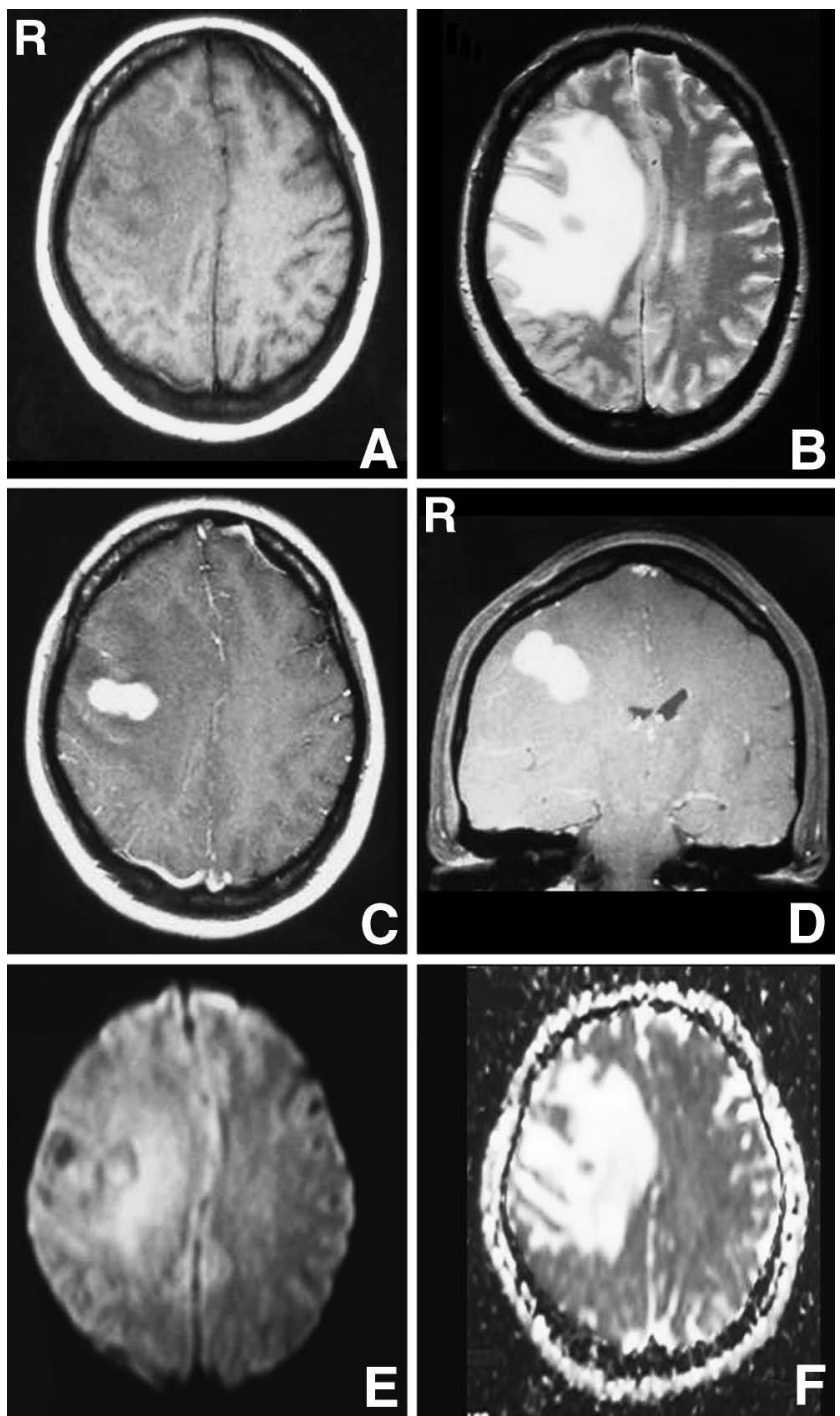

Fig. 1 Axial magnetic resonance (MR) images demonstrating a hypointense lesion on the $T_{1}$-weighted image $(A)$ and a hyperintense lesion on the $T_{2}$-weighted image (B) in the frontal lobe. Axial (C) and coronal (D) $T_{1}$-weighted MR images with gadolinium disclosing a well-defined mass with perifocal edema in the right frontal lobe. Diffusion-weighted MR image (E) showing a mild heterogeneous signal, and the corresponding apparent diffusion coefficient map ( $F$ ) revealing increased signal intensities. were normal except for raised leukocytosis (total white blood cell count $10.9 \times 10^{9}$ cells/l) and thrombocythemia (total platelet count $37.1 \times 10^{10} \mathrm{cells} / \mathrm{l}$ ). Radiography and computed tomography demonstrated no abnormality of the chest or abdominal organs. Magnetic resonance (MR) imaging study disclosed a lesion that appeared hypointense on $\mathrm{T}_{1}$-weighted and hyperintense on $\mathrm{T}_{2}$-weighted images, with homogeneous enhancement after gadolinium injection with significant surrounding edema in the right frontal lobe (Fig. 1). In addition, diffusion-weighted imaging showed a mild heterogeneous signal, and the corresponding apparent diffusion coefficient (ADC) maps revealed increased signal intensities. Internal carotid angiography demonstrated faint tumor staining in the capillary phase. The tentative preoperative diagnosis was malignant glioma, metastatic brain tumor, or malignant lymphoma.

The patient underwent fronto-parietal craniotomy with total removal of the tumor under motor cortex and tract monitoring of the motor evoked potentials. The tumor was dark gray in color, elastic, and had a well-defined cleavage plane. Examination of frozen sections indicated vasculitis or malignant lymphoma. Since the patient had temporary motor weakness of her face and upper limbs, and
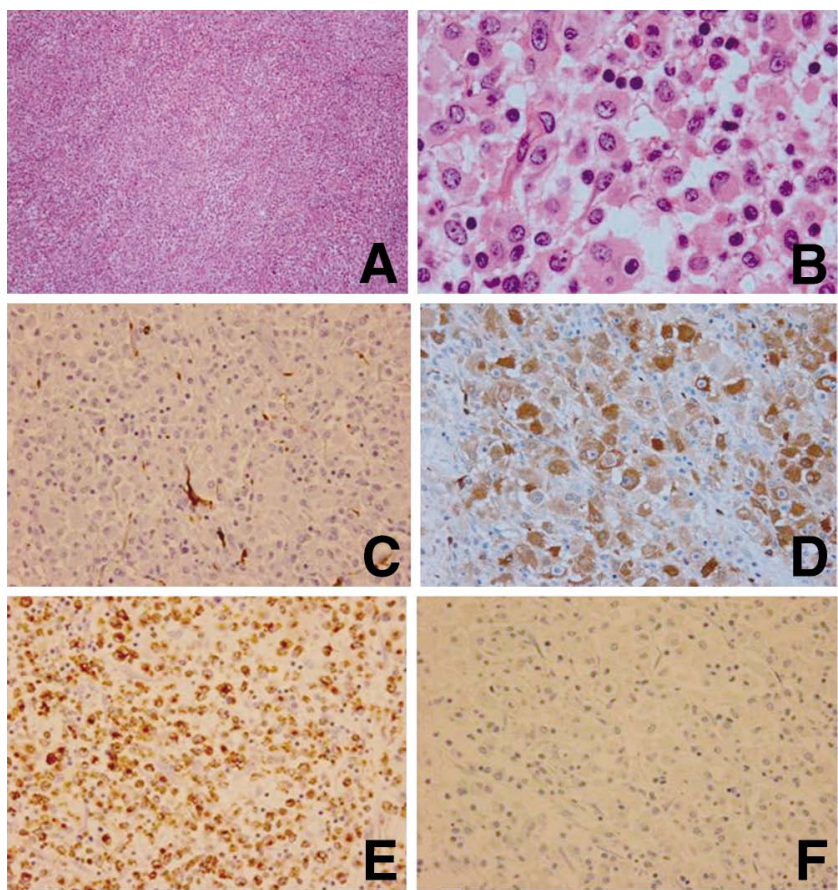

Fig. 2 A, B: Photomicrographs of the excised mass showing a polymorphous admixture of histiocytes, smaller lymphocytes and plasma cells, with the cytoplasm of the histiocytes containing intracytoplasmic lymphocytes and plasma cells (emperipolesis). Hematoxylin and eosin stain, original magnifications A: $\times 10$, B: $\times 320$. C-F: Photomicrographs of immunostaining showing the histiocytes were negative for glial fibrillary acidic protein (C), strongly positive for S-100 protein (D), strongly immunoreactive for CD68 (E), and negative for CD1a (F). Original magnifications $\times 160$. 
postoperative MR imaging showed moderate brain edema without enhancement, prednisone was administered (5 mg twice daily for 2 weeks). Subsequently, her symptoms disappeared within one month after the operation. After 5 months, physiological examination exhibited no symptoms, no lymphadenopathy, and no systemic masses, and follow-up MR imaging revealed no recurrence.

Histological examination demonstrated a polymorphous admixture of histiocytes, mature lymphocytes, and plasma cells without necrosis. These cellular infiltrates were set in a loose fibrous stroma. The lymphoplasmacytic cells were well differentiated, without atypical nuclei. The cytoplasm of some histiocytes was foamy and abundant, whereas the cytoplasm of others was pale and eosinophilic. The nuclei were round or oval, and exhibited a vesicular chromatin pattern with a single small nucleolus. The histiocytic cells displayed emperipolesis (lymphocyte phagocytosis), i.e., the presence of lymphocytes and plasma cells within the histiocyte cytoplasm. The blood vessels were often outlined by cuffs of lymphocytes and plasma cells. The histiocytes showed intense positive immunohistochemical staining for S-100 protein, CD68, smooth muscle actin, and vimentin, but were negative for cytokeratin, epithelial membrane antigen, glial fibrillary acidic protein, CD20, and CD1a. Based on these findings, the histological diagnosis was Rosai-Dorfman disease (Fig. 2).

\section{Discussion}

The present case of isolated intracranial Rosai-Dorfman disease occurred without dural attachment. Rosai-Dorfman disease or extranodal sinus histiocytosis with massive lymphadenopathy is an uncommon histiocytic proliferative disorder, and extranodal involvement, including the paranasal sinuses, skin, bone, and orbit, occurs in $43 \%$ of cases. ${ }^{4}$ Central nervous system (CNS) involvement is rare, and isolated intracranial Rosai-Dorfman disease is exceptionally rare, with approximately 70 reported cases, including the present case, and the characteristic features are distinct from those of the classical systemic disease. ${ }^{7,10,12,21,26)}$ The age of patients with isolated intracranial Rosai-Dorfman disease (mean 39.5 years) is greater than that of patients with the classical disease (mean 20.6 years)..$^{2,4,12,21)}$ Cervical lymphadenopathy is present in about $90 \%$ of patients with classical systemic RosaiDorfman disease, but rarely in patients with CNS involvement. The lesions can be solitary or multiple.

Most previously reported cases of intracranial RosaiDorfman disease showed dural attachment, and radiologically mimicked meningioma. Some cases were associated with adjacent bone erosion, which could lead physicians to consider a different diagnosis, such as metastasis, granulomatous disease, sarcoidosis, lymphoproliferative disorders, other histiocytoses, or in the case of suprasellar location, germinoma. On the other hand, only 6 isolated intraparenchymal cases without dural attachment, including the present case, have been reported (Table 1). ${ }^{1,6,11,17,18)}$ The masses were located in the frontal lobe in two cases, the parietal lobe in one, the temporal lobe in one, the cerebellum in one, and the fourth ventricle in one. Preoperative MR imaging demonstrated a homogeneous contrastenhanced lesion with significant surrounding edema in four previous cases. Such findings would commonly be considered to be consistent with malignant glioma, malignant lymphoma, or metastasis, and may lead to particular confusion, since MR imaging reveals a contrast-enhanced lesion with wide perifocal edema in both malignant lymphoma and benign cellular infiltration disease caused by inflammatory diseases such as Rosai-Dorfman disease.

In the present case, diffusion-weighted imaging of intracranial Rosai-Dorfman disease demonstrated mild heterogeneous signals on trace images, whereas the corresponding ADC maps showed increased signal intensities. These findings contrast with those of malignant lymphoma, which commonly appears as a homogeneous, strong signal on trace images and decreased signal intensity on ADC maps. The mechanism of Rosai-Dorfman disease may involve infiltration of small neoplastic cells such as malignant lymphoma, which disrupt the movement of water molecules, so vasogenic edema, brain tissue destruction, or infiltration of large cells, such as histiocytes may appear as increased intensity on ADC maps. Therefore, diffusion-weighted imaging findings may provide useful diagnostic information for identifying intracranial Rosai-Dorfman disease.

The symptoms of intracranial Rosai-Dorfman disease caused by the mass effect include headache, seizures, paralysis, and cranial nerve deficits. Rare cases with sellar

Table 1 Summary of patients with isolated intracranial Rosai-Dorfman disease without dural attachment

\begin{tabular}{|c|c|c|c|c|c|c|}
\hline $\begin{array}{l}\text { Case } \\
\text { No. }\end{array}$ & $\begin{array}{l}\text { Age (yrs)/ } \\
\text { Sex }\end{array}$ & Presentation & Location & $\begin{array}{l}\text { Preoperative } \\
\text { diagnosis }\end{array}$ & Treatment & Outcome \\
\hline 1 & $45 / \mathrm{F}$ & focal seizure & rt frontal lobe & glioma, meta. & resection & 5 mos, NED \\
\hline 2 & $22 / \mathrm{F}$ & diplopia & fourth ventricle & unknown & resection & 35 mos, NED \\
\hline 3 & $67 / F$ & cerebellar ataxia & rt cerebellar lobe & unknown & resection & $1 \mathrm{mo}, \mathrm{NED}$ \\
\hline 4 & $43 / \mathrm{F}$ & headache & rt parietal lobe & unknown & resection & 9 mos, NED \\
\hline 5 & $39 / \mathrm{M}$ & $\begin{array}{l}\text { vertigo, } \\
\text { visual disturbance }\end{array}$ & rt temporal lobe & meningioma & resection & 10 mos, NED \\
\hline $6^{*}$ & $33 / \mathrm{F}$ & headache & rt frontal lobe & glioma, meta. & $\begin{array}{l}\text { resection, } \\
\text { prednisone }\end{array}$ & 5 mos, NED \\
\hline
\end{tabular}

*Present case; others are cited from references 1, 6, 11, 17, and 18). F: female, M: male, meta.: metastatic brain tumor, NED: no evidence of disease, rt: right. 
lesions present with symptoms related to pituitary dysfunction and visual loss. ${ }^{19,27)}$ Previous intracranial RosaiDorfman disease cases most commonly revealed involvement of cervical lymph nodes (about half of cases), followed by the skeletal system, or salivary glands. ${ }^{4,23)}$

Most patients with Rosai-Dorfman disease follow a good clinical course; when the mass was surgically resected, the patients improved postoperatively. ${ }^{1,6,18)}$ However, relapse of intracranial Rosai-Dorfman disease has been encountered in some cases, despite surgical treatment. ${ }^{20}$ Moreover, some patients received corticosteroid treatment, anticancer agents, or radiotherapy, because of aggressive courses. ${ }^{5,8,10,25)}$ The mortality of patients with intracranial Rosai-Dorfman disease is approximately $7 \% .{ }^{14)}$ Low dose radiotherapy led to resolution of the residual mass after surgical resection. ${ }^{20}$ Stereotactic radiosurgery has also been used. A case with residual petroclival RosaiDorfman disease after surgical resection exhibited complete resolution following stereotactic radiosurgery. ${ }^{9)}$ Corticosteroid agents may also be an effective option in the treatment of patients with intracranial Rosai-Dorfman disease. One patient with multiple intracranial lesions, consistent with Rosai-Dorfman disease, was successfully treated with corticosteroid agents. ${ }^{15)}$ The pathophysiological findings suggest that Rosai-Dorfman disease is an expression of immune system dysfunction or possibly even an autoimmune process, indicating that corticosteroid treatment could be appropriate. ${ }^{2,16)}$ Epstein-Barr virus and human herpes virus type 6 have been detected by in situ hybridization in some specimens obtained from patients with Rosai-Dorfman disease. ${ }^{13)}$ However, the efficacy of such adjuvant therapy remains unclear, in view of the benign nature of the mass and the reports of spontaneous remission. Further studies on the cause and detailed mechanism of intracranial Rosai-Dorfman disease are needed.

The present extremely rare case of isolated intracranial Rosai-Dorfman disease without dural attachment indicates that Rosai-Dorfman disease should be considered in the differential diagnosis of intraparenchymal lesions appearing with enhancement on MR imaging with gadolinium.

\section{References}

1) Andriko JA, Morrison A, Colegial CH, Davis BJ, Jones RV: Rosai-Dorfman disease isolated to the central nervous system: a report of 11 cases. Mod Pathol 14: 172-178, 2001

2) Antonius JI, Farid SM, Baez-Giangreco A: Steroid-responsive Rosai-Dorfman disease. Pediatr Hematol Oncol 13: 563-570, 1996

3) Di Rocco F, Garnett MR, Puget S, Pueyerredon F, Roujeau T, Jaubert F, Sainte-Rose C: Cerebral localization of Rosai-Dorfman disease in a child. Case report. J Neurosurg 107(2 Suppl): 147-151, 2007

4) Foucar E, Rosai J, Dorfman R: Sinus histiocytosis with massive lymphadenopathy (Rosai-Dorfman disease): review of the entity. Semin Diagn Pathol 7: 19-73, 1990

5) Foucar E, Rosai J, Dorfman RF, Brynes RK: The neurologic manifestations of sinus histiocytosis with massive lymphadenopathy. Neurology 32: 365-372, 1982
6) Gaetani P, Tancioni F, Di Rocco M, Rodriguez Y, Baena R: Isolated cerebellar involvement in Rosai-Dorfman disease: case report. Neurosurgery 46: 479-481, 2000

7) Gupta DK, Suri A, Mahapatra AK, Mehta VS, Garg A, Sarkar C, Ahmad FU: Intracranial Rosai-Dorfman disease in a child mimicking bilateral giant petroclival meningiomas: a case report and review of literature. Childs Nerv Syst 22: 1194-1200, 2006

8) Haas RJ, Helmig MS, Prechtel K: Sinus histiocytosis with massive lymphadenopathy and paraparesis: remission with chemotherapy. A case report. Cancer 42: 77-80, 1978

9) Hadjipanayis CG, Bejjiani G, Wiley C, Hasegawa T, Maddock M, Kondziolka D: Intracranial Rosai-Dorfman disease treated with microsurgical resection and stereotactic radiosurgery. J Neurosurg 98: 165-168, 2003

10) Hinduja A, Aguilar LG, Steineke T, Nochlin D, Landolfi JC: Rosai-Dorfman disease manifesting as intracranial and intraorbital lesion. J Neurooncol 92: 117-120, 2009

11) Jurić G, Jakić-Razumović J, Rotim K, Zarković K: Extranodal sinus histiocytosis (Rosai-Dorfman disease) of the brain parenchyma. Acta Neurochir (Wien) 145: 145-149, 2003

12) Kidd DP, Revesz T, Miller NR: Rosai-Dorfman disease presenting with widespread intracranial and spinal cord involvement. Neurology 67: 1551-1555, 2006

13) Levine PH, Jahan N, Murari P, Manak M, Jaffe ES: Detection of human herpesvirus 6 in tissues involved by sinus histiocytosis with massive lymphadenopathy (Rosai-Dorfman disease). J Infect Dis 166: 291-295, 1992

14) McAlster WH, Herman I, Dehner LP: Sinus histiocytosis with massive lymphoadenopathy (Rosai-Dorfman disease). Pediatr Radiol 20: 425-432, 1990

15) McPherson CM, Brown J, Kim AW, Demonte F: Regression of intracranial Rosai-Dorfman disease following corticosteroid therapy. J Neurosurg 104: 840-844, 2006

16) Middel P, Hemmerlein B, Fayyazi A, Kaboth U, Radzum HJ: Sinus histiocytosis with massive lymphadenopathy: evidence for its relationship to macrophages and for a cytokinerelated disorder. Histopathology 35: 525-533, 1999

17) Morandi X, Godey B, Riffaud L, Heresbach N, Brassier G: Isolated Rosai-Dorfman disease of the fourth ventricle. Case illustration. J Neurosurg 92: 890, 2000

18) Natarajan S, Post KD, Strauchen J, Morgello S: Primary intracerebral rosai-dorfman disease: a case report. J Neurooncol 47: 73-77, 2000

19) Ng SB, Tan LH, Tan PH: Rosai-Dorfman disease of the breast: a mimic of breast malignancy. Pathology 32: 10-15, 2000

20) Petzold A, Thom M, Powell M, Plant GT: Relapsing intracranial Rosai-Dorfman disease. J Neurol Neurosurg Psychiatry 71: 538-541, 2001

21) Purav P, Ganapathy K, Mallikarjuna VS, Annapurneswari S, Kalyanaraman S, Reginald J, Natarajan P, Bapu KR, Balamurugan M: Rosai-Dorfman disease of the central nervous system. Clin Neurosci 12: 656-659, 2005

22) Rosai J, Dorfman RF: Sinus histiocytosis with massive lymphadenopathy. A newly recognized benign clinicopathological entity. Arch Pathol 87: 63-70, 1969

23) Sakai K, Koike G, Seguchi K, Nakazato Y: Sinus histiocytosis with massive lymphadenopathy: a case of multiple dural involvement. Brain Tumor Pathol 15: 63-69, 1998

24) Sharma MS, Padua MD, Jha AN: Rosai-Dorfman disease mimicking a sphenoid wing meningioma. Neurol India 53: 110-111, 2005

25) Shaver EG, Rebsamen SL, Yachnis AT, Sutton LN: Isolated extranodal intracranial sinus histiocytosis in a 5-year-old 
boy. Case report. J Neurosurg 79: 769-773, 1993

26) Wan S, Teng X, Zhan R, Yu J, Gu J, Zhang K: Isolated intracranial Rosai-Dorfman disease mimicking suprasellar meningioma: case report with review of the literature. J Int Med Res 36: 1134-1139, 2008

27) Woodcock RJ Jr, Mandell JW, Lipper MH: Sinus histiocytosis (Rosai-Dorfman disease) of the suprasellar region: MR imaging findings—a case report. Radiology 213: 808-810,
1999

Address reprint requests to: Takao Fukushima, MD, PhD, Department of Neurological Surgery, Nihon University School of Medicine, 30-1 Ohyaguchi-kamimachi, Itabashi-ku, Tokyo 173-8610, Japan.

e-mail: fukushima.takao@nihon-u.ac.jp 